\title{
BPIFB1 loss alters airway mucus properties and diminishes mucociliary clearance
}

Lauren J. Donoghue ${ }^{1,2}$, Matthew R. Markovetz ${ }^{3}$, Cameron B. Morrison ${ }^{3}$, Kathryn M. McFadden ${ }^{1}$, Troy D. Rogers ${ }^{3}$, Brian Button ${ }^{3,4}$, Camille Ehre ${ }^{3,5}$, David B. Hill ${ }^{3,6}$, Barbara R. Grubb ${ }^{3}$, and Samir N. P. Kelada ${ }^{1,3^{*}}$

${ }^{1}$ Department of Genetics, University of North Carolina at Chapel Hill

${ }^{2}$ Curriculum in Genetics and Molecular Biology, University of North Carolina at Chapel Hill

${ }^{3}$ Marsico Lung Institute, University of North Carolina at Chapel Hill

${ }^{4}$ Department of Biochemistry and Biophysics, University of North Carolina at Chapel Hill

${ }^{5}$ Division of Pediatric Pulmonology, University of North Carolina at Chapel Hill

${ }^{6}$ Department of Physics and Astronomy, University of North Carolina at Chapel Hill

*Corresponding author

Samir Kelada, PhD MPH

University of North Carolina

Department of Genetics

120 Mason Farm Road

Chapel Hill, NC 27599

919-962-2148

samir_kelada@med.unc.edu

Conflict of interest statement: "The authors have declared that no conflict of interest exists." 


\section{$1 \quad$ ABSTRACT}

2 Airway mucociliary clearance (MCC) is required for host defense and often diminished in

3 chronic lung diseases. Effective clearance depends upon coordinated actions of the airway

4 epithelium and a mobile mucus layer. Dysregulation of the primary secreted airway mucin

5 proteins, MUC5B and MUC5AC, is associated with a reduction in the rate of MCC; however,

6 how other secreted proteins impact the integrity of the mucus layer and MCC remains unclear.

7 We previously identified the gene Bpifb1/Lplunc1 as a regulator of airway MUC5B levels using

8 genetic approaches. Here, we show that BPIFB1 is required for normal mucociliary clearance in

9 vivo using Bpifb1 knockout (KO) mice. Reduced MCC in Bpifb1 KO mice occurred in the absence

10 of defects in sodium or chloride ion transport or reduced ciliary beat frequency. BPIFB1 loss

11 resulted in airway mucus flakes with significantly increased complex viscosity, a key biophysical

12 property of mucus known to impact MCC. Finally, we detected colocalization of BPIFB1 and

13 MUC5B in secretory granules in mice and in the protein mesh of secreted mucus in human

14 airway cultures. Collectively, our findings demonstrate that BPIFB1 is an important component

15 of the mucociliary apparatus in mice and a key component of the mucus protein network.

\section{INTRODUCTION}

18 The airway mucus layer plays a critical role in airway homeostasis and host defense in part by

19 facilitating mucociliary clearance (MCC) of inhaled particles and pathogens. Effective MCC

20 requires a properly hydrated airway surface, an optimal balance of components in secreted

21 mucus (i.e., proteins, salt), and coordinated beating of cilia. The importance of the MCC

22 apparatus is highlighted by the fact that mutations in genes essential to ciliary function or ion 
transport cause primary ciliary dyskinesia (PCD) and cystic fibrosis (CF), respectively, in which respiratory infections are prominent and severe. In the context of more common obstructive airway disease such as asthma and chronic obstructive pulmonary disease (COPD), changes in MCC have been associated with variation in the abundance of the secreted mucin proteins MUC5B and MUC5AC, which are key contributors to the gel-like nature of healthy airway mucus $^{1}$. The concentration, organization, and post-translational modifications of MUC5B and MUC5AC also have been shown to alter biophysical properties of mucus that are required for effective $\mathrm{MCC}^{2-6}$. Furthermore, the ability of mucins to form a proper gel-structure is dependent upon interactions with other secreted proteins, referred to as the "mucin interactome" ${ }^{\prime 7}$, although the specifics of these dependencies remain unclear. is constitutively expressed in the airways of healthy individuals where it has a role in regulating homeostasis through airway MCC and host defense ${ }^{8,9}$. We previously identified the gene Bpifb1 (also known as Lplunc1) as a regulator of airway MUC5B protein levels using a quantitative genetics approach in mice and showed that loss of BPIFB1 in a knockout (KO) mouse model led to increased levels of MUC5B protein in the airways ${ }^{10}$. Given that MUC5B levels have been shown to affect $\mathrm{MCC}^{9,11}$, we sought to determine if Bpifb1 also had a role in regulating MCC. We observed a substantial loss of MCC in the lower airways of Bpifb1 KO mice. To identify

42 which aspects of the MCC apparatus contributed to this defect, we assessed epithelial sodium and chloride ion transport, ciliary function, and biophysical properties of mucus. Our results 
associated with decreased MCC in the absence of BPIFB1 and that BPIFB1 is a component of the mucus protein network.

\section{RESULTS AND DISCUSSION}

In order to determine if Bpifb1 has a role in MCC, we assessed MCC in tracheas of naïve Bpifb1

KO and WT mice. We quantified MCC by measuring the percentage of instilled beads that were

1A). These results indicated clearly that BPIFB1 is required for MCC.

To understand the mechanism by which BPIFB1 loss leads to defective MCC, we systematically tested the expression and function of key components of the MCC apparatus. First, we examined the expression of key ion transporters in the airway epithelium given their importance in regulating airway hydration and $\mathrm{MCC}^{1}$. RNA sequencing of lung tissue from sodium and chloride ion transporter genes including Cftr, Scnn1b, and Tmem16a (Figure S1).

61 However, given that a lack of difference in ion transporter gene expression does not rule out a

62 difference in functionality, we also measured bioelectric capacities in Bpifb1 KO and WT tracheas. We found no differences in short-circuit current $\left(I_{s c}\right)$ at baseline or following

64 stimulation with compounds targeting the major ion channels in the airway epithelium (i.e. CFTR, ENaC, CaCC), (Figure 1B). This indicated that the loss of MCC in Bpifb1 KO mice was likely 
67 directly measured ciliary beat frequency (CBF) in intact tracheas in situ. In comparison to WT

68

69

70

71

72

73

74

mice, Bpifb1 KO mice had a slightly elevated CBF, albeit not statistically significant at $P<0.05$

(Fig 1C). These data indicated that reduced MCC was not associated with reduced ciliary beating.

Next, to determine if Bpifb1 loss influenced the expression of other genes or biological processes that could underlie the defect in MCC, we examined global gene expression in lung tissue from Bpifb1 KO and WT mice. Excluding Bpifb1, only 4 protein coding genes and 2 long non-coding RNAs were differentially expressed by genotype ( fold change $\mid \geq 2$ and adjusted $P$ value $\leq 0.05$, Figure S1). Of these genes, none have been previously annotated for a direct role in MCC or related pathways. Furthermore, genes related to mucin production, including Muc5b, Muc5ac, Spdef, and Foxa3, were not differentially expressed. These gene expression results suggested the reduction in MCC in Bpifb1 KO mice was not mediated by differential expression of key MCC or mucus production genes.

Given the lack of differences in hallmark causes of reduced MCC, we investigated if there were differences in the airway mucus per se that could be contributing to the observed loss of MCC. Using microbead rheology, we measured the complex viscosity $\left(\eta^{*}\right)$, a measure of both viscosity and elasticity, of mucus flakes (i.e. the insoluble portions of airway mucus) in bronchoalveolar lavage (BAL) fluid from Bpifb1 KO vs WT mice. For these measurements, mice were intranasally exposed to house dust mite allergen for three weeks to increase the levels of mucus in BAL, and there were no differences in the degree of eosinophilic inflammation by genotype 
89 (Supplementary Figure S2). We found that the complex viscosity of mucus flakes was significantly increased in Bpifb1 KO mice compared to WT mice (Figure 2A, B). By imaging of

91 whole BAL, we determined this increase was independent of the size and number of mucus

92 flakes, and there were no differences in these metrics by genotype (Supplementary Figure S3A-

E). Consistent with these findings and our previous report of increased MUC5B in BAL from

94 naïve Bpifb1 KO mice ${ }^{10}$, we found that MUC5B levels were elevated by genotype in the

95 supernatant fraction of centrifuged BAL from naïve mice, but not in the pelleted (i.e. insoluble)

96 fraction (Figure 2C). Thus, loss of BPIFB1 results in altered biophysical properties of mucus

97 flakes and increased levels of soluble MUC5B.

To gain insight into whether BPIFB1 colocalizes with mucin protein in the airways and, if so,

whether this association occurs before mucin granule release, we assessed the spatial

101 relationship between BPIFB1 and mucin protein using fluorescent immunostaining. By

102 colocalization analysis of labeled BPIFB1 and MUC5B protein in large airways of mice challenged

103 with allergen, we determined that the vast majority of MUC5B positive cells were also positive

104 for BPIFB1 (Figure 3A, B), indicating regulated cellular co-expression of MUC5B and BPIFB1.

105 Colocalization was also present in the airways of mice not challenged with allergen

106 (Supplementary Figure S4). Furthermore, MUC5B positive granules were also positive for

107 BPIFB1 and luminal mucus contained both MUC5B and BPIFB1 (Figure 3C), suggesting that

108 packaging and secretion of these proteins is likely coupled. 
110 Finally, we performed immunogold-labeled scanning electron microscopy for BPIFB1 and

111 MUC5B in human bronchial epithelial cells (HBECs) cultured at air-liquid-interface to determine

112 if BPIFB1 closely associated with extracellular mucus. We detected BPIFB1 exclusively in mucus

113 strands coating the cellular surface (Figure 4). This finding confirmed that BPIFB1 is a

114 component of the mucus protein network produced by human airway cells.

116 Significantly altered levels of either BPIFB1 gene or protein expression have been observed in

$117 \mathrm{CF}, \mathrm{COPD}$, asthma, and $\mathrm{PPF}^{12-15}$, indicating a strong connection between BPIFB1 and muco-

118 obstructive diseases. After identifying Bpifb1 as a regulator of airway mucus levels through an

119 unbiased genome-wide search in the mouse ${ }^{10}$, we sought to interrogate the specific function of

120 BPIFB1 in the airways. We found that the loss of BPIFB1 reduced MCC dramatically in naïve

121 Bpifb1 KO mice, demonstrating the critical role of this gene in regulating MCC. Thus, in addition

122 to the strong connection between BPIFB1 gene/protein expression and muco-obstructive

123 diseases, our findings indicate BPIFB1 may have an important role in airway host defense by

124 regulating MCC. Studies in humans have shown that there is significant interindividual variation

125 in baseline MCC, and analysis of MCC in monozygotic and dizygotic twins has implicated genetic

126 variation as a modifier of $\mathrm{MCC}^{16}$. Given the identification of common genetic variation

127 associated with the levels of BPIFB1 gene expression ${ }^{17}$, genetic variation in BPIFB1 regulation

128 could be one such factor regulating baseline MCC in humans. Furthermore, BPIFB1 was one of

129 the top 20 proteins detected in endotracheal tube mucus from individuals without a history of

130 lung disease or smoking, highlighting its abundance in non-disease states ${ }^{18}$. 
132 Due to the lack of differences in basal or stimulated transport of sodium and chloride ions,

133 ciliary beating, and MCC-related gene expression between Bpfib1 KO and WT mice, our data

134 point toward changes to the airway mucus layer as the cause of reduced MCC. More

135 specifically, the increased complex viscosity of mucus, as we observed in mucus flakes from

136 Bpifb1 KO mice and may be the case for soluble mucus as well, is suggestive of mucus that is

137 more difficult to clear from the airways. In vitro studies with human airway cultures ${ }^{19-22}$, as well

138 as earlier studies in model organisms ${ }^{23-25}$, have demonstrated that mucus transport is directly

139 related to the rheological properties of mucus. The increased CBF that we observed in Bpifb1

140 KO mice, although not statistically significant, may be indicative of an attempt to compensate

141 for altered airways mucus ${ }^{26}$. Such a phenomenon has been demonstrated in human bronchial

142 epithelial cultures where CBF increased following a moderate increase in mucus viscosity or

143 mucus load 27.

145 The increased complex viscosity of mucus flakes in Bpifb1 KO mice may be attributable to a

146 change in mucin network structure, which is a function of the density and/or nature of mucin-

147 mucin/mucin-protein interactions ${ }^{7}$. In muco-obstructive airway disease contexts, for example,

148 heightened mucus viscoelasticity due to oxidation-dependent mucin cross-linking ${ }^{5}$ or increased

149 mucin concentration ${ }^{28}$ has been described. Direct measurement of mucin concentration was

150 not possible in our experiments due to the high dilution factor of mucus in lavage fluid.

151 However, the lack of increased MUC5B in pelleted BAL from Bpifb1 KO mice provides some

152 evidence against the hypothesis that mucin concentration is increased in mucus flakes and is

153 the driver of changes in mucus rheology. That said, we detected elevated levels of soluble 
154 MUC5B in Bpifb1 KO mice, which raises the question of whether MUC5B levels are higher in

155 Bpifb1 KO mice simply due to decreased MCC or increased mucin concentration. In vitro

156 studies, in which mucus can be collected directly from the surface of epithelial cells, will be

157 required to determine specifically how BPIFB1 impacts the mucus layer.

159 Although the polymeric nature of gel-forming mucins is essential for the biophysical properties

160 of mucus, studies have shown that mucins alone cannot create complex networks, and thus,

161 other factors, including secreted globular proteins, are necessary for mucus formation ${ }^{29}$. Our

162 detection of BPIFB1 in secreted mucus from HBECs confirms BPFIB1 is indeed a component of

163 the mucus protein network and supports the conclusion that this non-mucin protein plays an

164 important role in the mucus layer. Further evidence for a direct role of BPIFB1 in the mucus

165 network comes from a finding that BPIFB1 has strong physical interactions with mucin proteins

166 in HEBC secretions. Radicioni et al. found that after treating mucus from HBEC cultures with

167 guanidinium hydrochloride and detergents (i.e. dissociative conditions), BPIFB1 was one of few

168 globular proteins interacting with mucins ${ }^{7}$. Additionally, our data demonstrating shared

169 regulatory mechanisms between BPIFB1 and MUC5B suggest strongly coordinated roles. We

170 previously found that Bpifb1 and Muc5b gene expression was strongly correlated in mouse lung

171 tissue ${ }^{10}$, and here we have shown that BPIFB1 and MUC5B protein are colocalized in airway

172 epithelial cells and present in secreted mucus. Cellular co-expression between BPIFB1 and

173 MUC5B mRNA has also been demonstrated in human airways by single cell RNA sequencing

174 studies $^{30,31}$ and between BPIFB1 and MUC5AC protein in COPD ${ }^{32}$. Lastly, our finding that BPIFB1

175 is present in mucin secretory granules, which are tightly packed and only contain the necessary 
176 proteins for mucus, implicates an additional regulatory mechanism coupling these two proteins.

177 Whether there is an optimal stoichiometry between levels of secreted BPIFB1 and mucin

178 proteins for MCC, as may be the case between MUC5B and MUC5AC 2,33 , remains to be

179 determined.

180

181 In summary, we demonstrate that BPIFB1 is critical for MCC in vivo. These findings establish

182 that mucociliary dysfunction can be driven by non-mucin protein components of airway mucus,

183 highlighting their importance in health and disease.

186 METHODS

187 A detailed descriptions of the methods used in this study can be found in the Supplementary

188 Methods (Supplementary File 1).

\section{Study Approval.}

191 All experiments conducted with mice in this study were compliant with an Institutional Animal

192 Care and Use Committee protocol at an animal facility approved and accredited by the

193 Association for Assessment and Accreditation of Laboratory Animal Care International. Primary

194 cells were provided by the Cystic Fibrosis (CF) Center Tissue Core Facility of the University of

195 North Carolina at Chapel Hill under the auspices of protocols approved by the Institutional

196 Committee on the protection of the rights of human subjects. 
Data availability. Complete raw data and counts generated by RNA sequencing were deposited in the NCBI's Gene Expression Omnibus (GEO) database under entry GSE150452.

\section{AUTHOR CONTRIBUTIONS}

LJD, MRM, CBM, BB, CE, DBH, BRG, and SNPK conceived experiments and interpreted data. LJD, the manuscript. All authors edited and reviewed the manuscript.

\section{ACKNOWLEDGEMENTS}

We thank Aiman Abzhanova for assistance with immunostaining. This work was supported in

211 National Institute of General Medical Sciences under award 5T32 GM007092.

212

213

214

215

\section{REFERENCES}

1. Fahy J V., Dickey BF. Airway mucus function and dysfunction. N Engl J Med. 2011;364(10):978. doi:10.1056/NEJMc1014719

2. Kesimer M, Ford AA, Ceppe A, et al. Airway mucin concentration as a marker of chronic bronchitis. N Engl J Med. 2017;377(10):911-922. doi:10.1056/NEJMoa1701632

3. Bonser LR, Zlock L, Finkbeiner W, Erle DJ. Epithelial tethering of MUC5AC-rich mucus impairs mucociliary transport in asthma. J Clin Invest. 2016;126(6):2367-2371. 
doi:10.1172/JCl84910

222 4. Kesimer M, Makhov AM, Griffith JD, Verdugo P, Sheehan JK. Unpacking a gel-forming mucin : a view of MUC5B organization after granular release. 2018:15-22. doi:10.1152/ajplung.00194.2009.

5. Yuan S, Hollinger M, Lachowicz-Scroggins ME, et al. Oxidation increases mucin polymer cross-links to stiffen airway mucus gels. Sci Transl Med. 2015;7(276):1-10. doi:10.1126/scitrans/med.3010525

6. Welsh KG, Rousseau K, Fisher G, et al. MUC5AC and a Glycosylated Variant of MUC5B Alter Mucin Composition in Children With Acute Asthma. Chest. 2017;152(4):771-779. doi:10.1016/j.chest.2017.07.001

7. Radicioni G, Cao R, Carpenter J, et al. The innate immune properties of airway mucosal surfaces are regulated by dynamic interactions between mucins and interacting proteins: the mucin interactome. Mucosal Immunol. 2016;9(6):1442-1454. doi:10.1038/mi.2016.27

8. Roy MG, Livraghi-Butrico A, Fletcher AA, et al. Muc5b is required for airway defence.

Nature. 2014;505(7483). doi:10.1038/nature12807

9. Hancock LA, Hennessy CE, Solomon GM, et al. Muc5b overexpression causes mucociliary dysfunction and enhances lung fibrosis in mice. Nat Commun. 2018;9(1):1-10. doi:10.1038/s41467-018-07768-9

10. Donoghue L, Livraghi-Butrico A, McFadden K, et al. Identification of trans Protein QTL for 
11. Evans CM, Raclawska DS, Ttofali F, et al. The polymeric mucin Muc5ac is required for allergic airway hyperreactivity. Nat Commun. 2015;6(May 2014). doi:10.1038/ncomms7281

12. Bingle $\mathrm{L}$, Wilson $\mathrm{K}$, Musa $\mathrm{M}$, et al. BPIFB1 (LPLUNC1) is upregulated in cystic fibrosis lung disease. Histochem Cell Biol. 2012;138(5):749-758. doi:10.1007/s00418-012-0990-8

13. Gao J, Ohlmeier S, Nieminen P, et al. Elevated sputum BPIFB1 levels in smokers with chronic obstructive pulmonary disease: a longitudinal study. Am J Physiol Lung Cell Mol Physiol. 2015;309(1):L17-26. doi:10.1152/ajplung.00082.2015

14. Wu J, Kobayashi M, Sousa EA, et al. Differential Proteomic Analysis of Bronchoalveolar Lavage Fluid in Asthmatics following Segmental Antigen Challenge. Mol Cell Proteomics. 2005;4(9):1251-1264. doi:10.1074/mcp.M500041-MCP200

15. Bingle CD, Araujo B, Wallace $\mathrm{W}$ a, Hirani N, Bingle L. What is top of the charts? BPIFB1/LPLUNC1 localises to the bronchiolised epithelium in the honeycomb cysts in UIP. Thorax. 2013;68(12):1167-1168. doi:10.1136/thoraxjnl-2013-204179

16. Camner P, Philipson K, Friberg L. Tracheobronchial Clearance in Twins. 1972:Vol 24.

17. Saferali A, Obeidat $M$, Bérubé J-C, et al. Polymorphisms associated with expression of BPIFA1/BPIFB1 and lung disease severity in cystic fibrosis. Am J Respir Cell Mol Biol. 2015;53(5):607-614. doi:10.1165/rcmb.2014-01820C

18. Markovetz MR, Subramani DB, Kissner WJ, et al. Endotracheal tube mucus as a source of airway mucus for rheological study. Am J Physiol Lung Cell Mol Physiol. 2019;317(4):L498L509. doi:10.1152/ajplung.00238.2019

19. King M. Experimental models for studying mucociliary clearance. Eur Respir J. 
1998;11(1):222-228. doi:10.1183/09031936.98.11010222

266

267

268

269

270

271

272

273

274

275

276

277

278

279

280

281

282

283

284

20. Hill DB, Vasquez PA, Mellnik J, et al. A Biophysical Basis for Mucus Solids Concentration as a Candidate Biomarker for Airways Disease. 2014;9(2):1-11. doi:10.1371/journal.pone.0087681

21. Button B, Cai LH, Ehre C, et al. A periciliary brush promotes the lung health by separating the mucus layer from airway epithelia. Science (80- ). 2012;337(6097):937-941. doi:10.1126/science.1223012

22. Carpenter J, Lynch SE, Cribb JA, Kylstra S, Hill DB, Superfine R. Buffer drains and mucus is transported upward in a tilted mucus clearance assay. Am J Physiol - Lung Cell Mol Physiol. 2018;315(5):L910-L918. doi:10.1152/ajplung.00274.2018

23. Shih CK, Litt M, Khan MA, Wolf DP. Effect of nondialyzable solids concentration and viscoelasticity on ciliary transport of tracheal mucus. Am Rev Respir Dis. 1977;115(6):989995. doi:10.1164/arrd.1977.115.6.989

24. Lorenzi G, Bohm GM, Guimaraes ET, Costa Vaz MA, King M, Saldiva PHN. Correlation between rheologic properties and in vitro ciliary transport of rat nasal mucus. Biorheology. 1992;29(4):433-440. doi:10.3233/BIR-1992-29406

25. Yu DM, Amidon GL, Weiner ND, Fleisher D, Goldberg AH. The Role of Rheological Properties in Mucociliary Transport by Frog Palate Ciliated Model. Pharm Res. $1994 ; 11(12)$.

26. Button B, Okada SF, Frederick CB, Thelin WR, Boucher RC. Mechanosensitive ATP release maintains proper mucus hydration of airways. Sci Signal. 2013;6(279):1-10. doi:10.1126/scisignal.2003755 
287

27. Liu L, Shastry S, Byan-Parker S, et al. An autoregulatory mechanism governing mucociliary transport is sensitive to mucus load. Am J Respir Cell Mol Biol. 2014;51(4):485-493. doi:10.1165/rcmb.2013-0499MA

28. Esther CR, Muhlebach MS, Ehre C, et al. Mucus accumulation in the lungs precedes structural changes and infection in children with cystic fibrosis. Sci Transl Med. 2019;11(486). doi:10.1126/scitransImed.aav3488

29. Thornton DJ, Sheehan JK. From mucins to mucus: toward a more coherent understanding of this essential barrier. Proc Am Thorac Soc. 2004;1(1):54-61. doi:10.1513/pats.2306016

30. Braga FAV, Kar G, Berg M, et al. Cell States in Health and in Asthma. Nat Med. 2019.

31. Goldfarbmuren KC, Jackson ND, Sajuthi SP, et al. Dissecting the cellular specificity of smoking effects and reconstructing lineages in the human airway epithelium. Nat Commun. 2020;11(1). doi:10.1038/s41467-020-16239-z

32. De Smet EG, Seys LJM, Verhamme FM, et al. Association of innate defense proteins BPIFA1 and BPIFB1 with disease severity in COPD. Int J COPD. 2018;13:11-27. doi:10.2147/COPD.S144136

33. Kirkham S, Kolsum U, Rousseau K, Singh D, Vestbo J, Thornton DJ. MUC5B is the major mucin in the gel phase of sputum in chronic obstructive pulmonary disease. Am J Respir Crit Care Med. 2008;178(10):1033-1039. doi:10.1164/rccm.200803-3910C 
FIGURE LEGENDS

Figure 1. Decreased mucociliary clearance in Bpifb1 KO mice. (A) Percent bead clearance in tracheal mucociliary clearance assay in naive WT and Bpifb1 KO mice. Mean \pm SEM depicted. $\mathrm{n}=6 /$ genotype. Significance of two-sided Student's $t$-test denoted as $* *, P<0.01$. (B) Ion

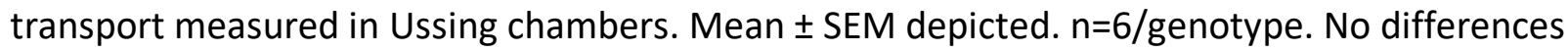
between genotypes by two-sided Student's $t$-test. (C) Ciliary beat frequency by in situ tracheal assay. Mean \pm SEM depicted. $n=7-8 /$ genotype. Difference between genotypes not significant by two-sided Welch's $t$-test, $\mathrm{P}=0.1$.

Figure 2. Biophysical properties of airway mucus are altered in Bpifb1 KO mice.

(A) Distribution of rheological signal in BAL from a representative allergen exposed WT and interquartile ranges. $n=13 /$ genotype. Significant difference between genotypes by ANCOVA modeling denoted as *, $P<0.05$. (C) MUC5B in pellet and supernatant fractions of BAL from naive WT and Bpifb1 KO mice by mucin western blotting. Mean \pm SEM depicted. Significant difference between genotypes in supernatant fraction by two-sided Welch's $t$-test denoted as $* *, P<0.01 . \mathrm{n}=5 /$ genotype.

328 and MUC5B (red) immunostaining in airway epithelial cells from allergen challenged mice. Scale bar $=10 \mu \mathrm{m}$. (B) Percentage of cells positively stained for BPIFB1 only, MUC5B only, or both in allergen challenged mice. $n=7$ mice. (C) BPIFB1 (green) and MUC5B (red) immunostaining in 
331 large airways of allergen challenged mice demonstrating BPIFB1 staining in luminal mucus.

332 Scale bar $=50 \mu \mathrm{m}$.

333

334 Figure 4: BPIFB1 is part of mucus network in human bronchial epithelial cell cultures.

335 Immunogold-labeled scanning electron microscopy for (A) negative control, (B) MUC5B, and (C)

336 BPIFB1. Scale bar $=1 \mu \mathrm{m}$.

337

338 Supplementary Figure Legends provided in Supplementary File 1.

339

340 


\section{A}

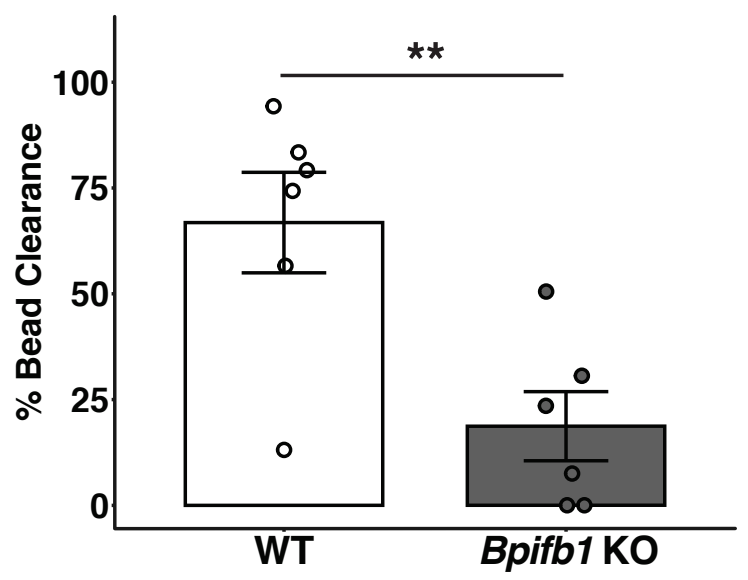

B

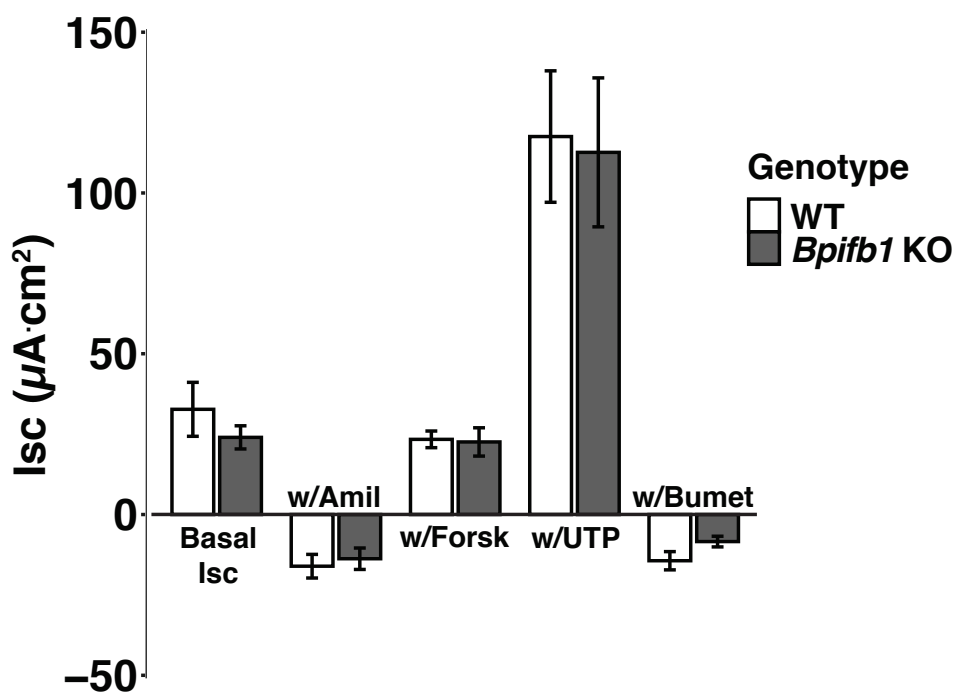

C

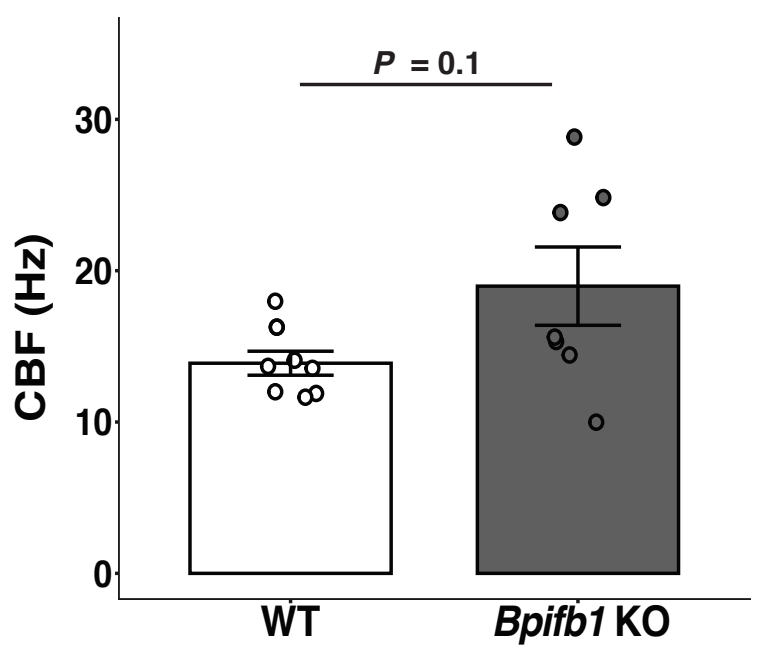

Figure 1. Decreased mucociliary clearance in Bpifb1 KO mice.

(A) Percent bead clearance in tracheal mucociliary clearance assay in naive WT and Bpifb1 KO mice. Mean \pm SEM depicted. $n=6 / g e n o t y p e$. Significance of two-sided Student's $t$-test denoted as ${ }^{* *}, P<0.01$. (B) lon transport measured in Ussing chambers. Mean \pm SEM depicted. $n=6 / g e n o t y p e$. No differences between genotypes by two-sided Student's $t$-test. (C) Ciliary beat frequency by in situ tracheal assay. Mean \pm SEM depicted. $n=7-8 /$ genotype. Difference between genotypes not significant by two-sided Welch's $t$-test, $P=0.1$. 
A

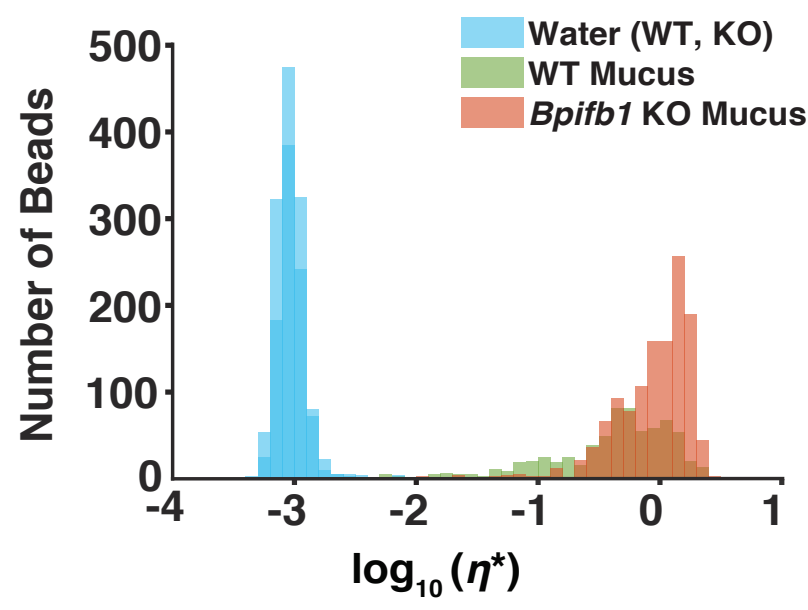

B

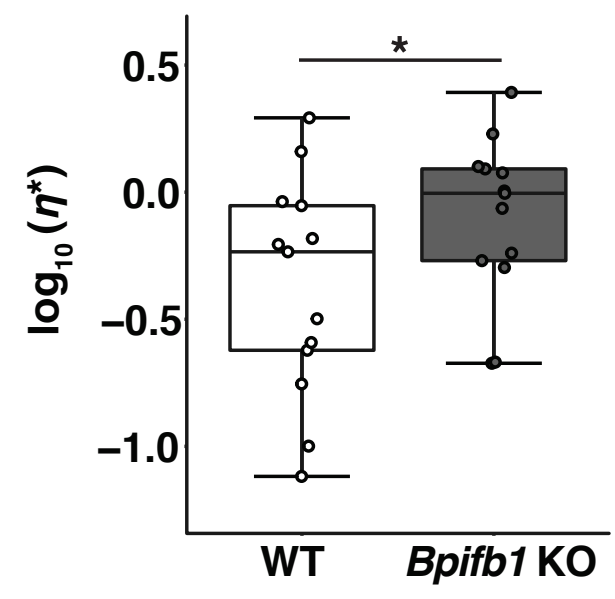

C

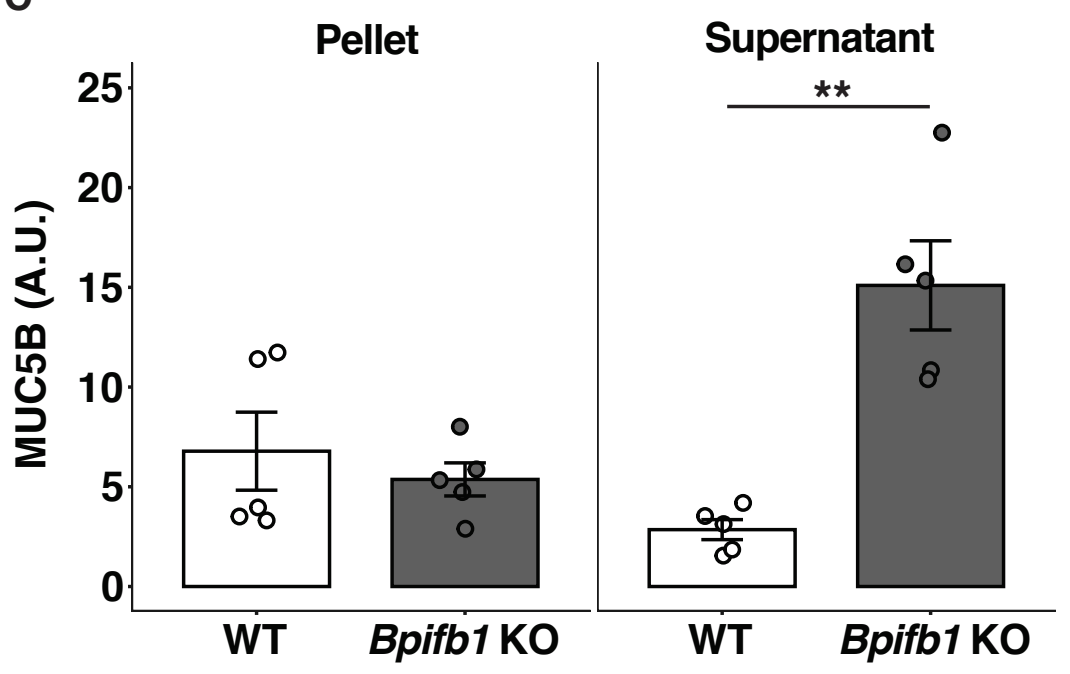

Figure 2. Biophysical properties of airway mucus are altered in Bpifb1 KO mice.

(A) Distribution of rheological signal in BAL from a representative allergen exposed WT and Bpifb1 KO mouse. (B) Complex viscosity of BAL mucus fraction. Boxplots depict median and interquartile ranges. $n=13 / g e n o t y p e$. Significant difference between genotypes by ANOVA modeling denoted as ${ }^{*}, P<0.05$. (C) MUC5B in pellet and supernatant fractions of BAL from naive WT and Bpifb1 KO mice by mucin western blotting. Mean \pm SEM depicted. Significant difference between genotypes in supernatant fraction by two-sided Welch's $t$-test denoted as ${ }^{* *}, P<0.01$. $\mathrm{n}=5 /$ genotype. 
A
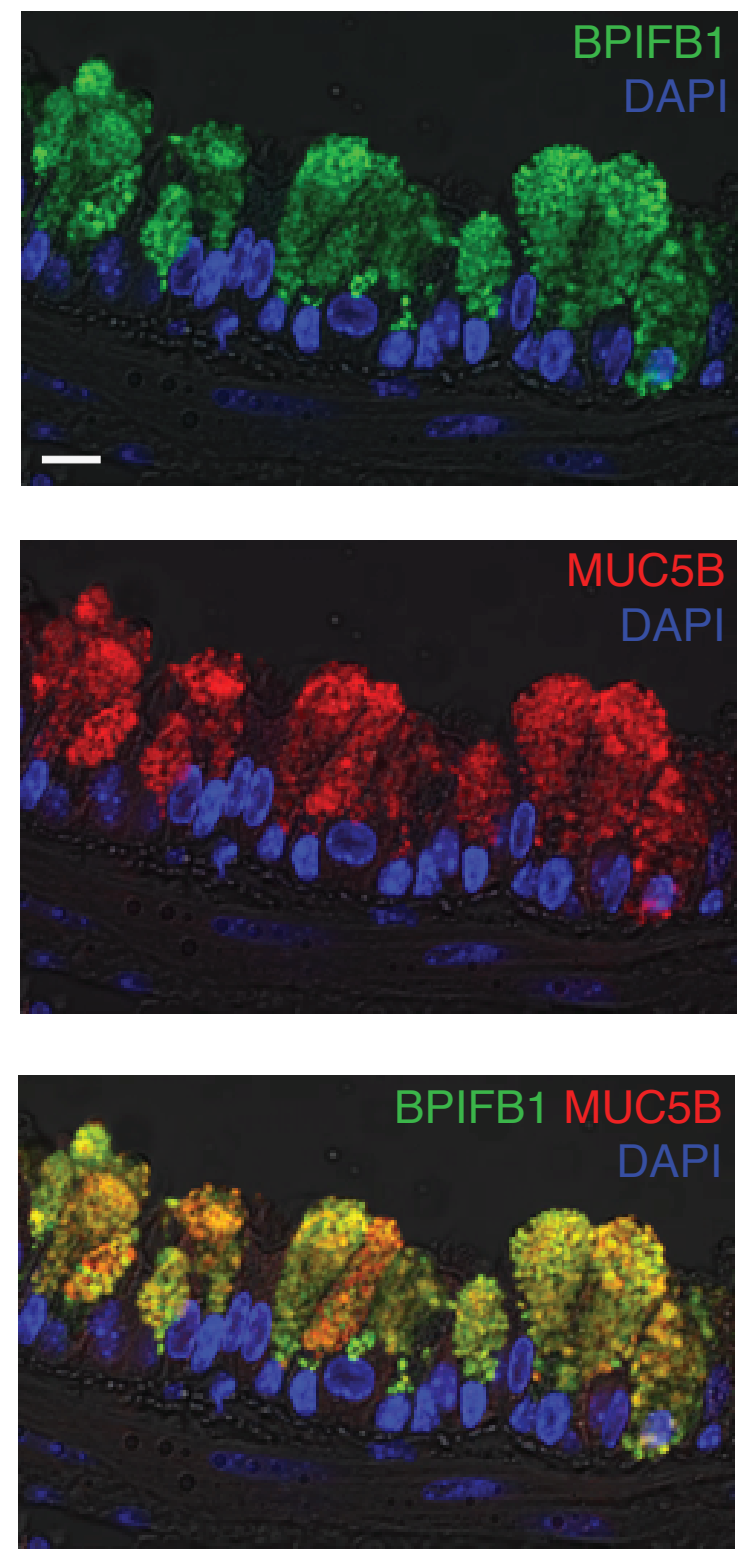

B

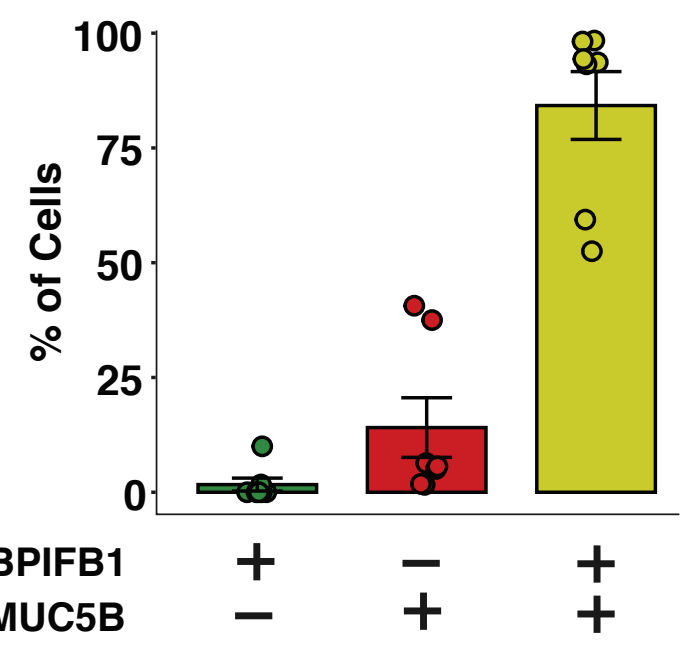

C
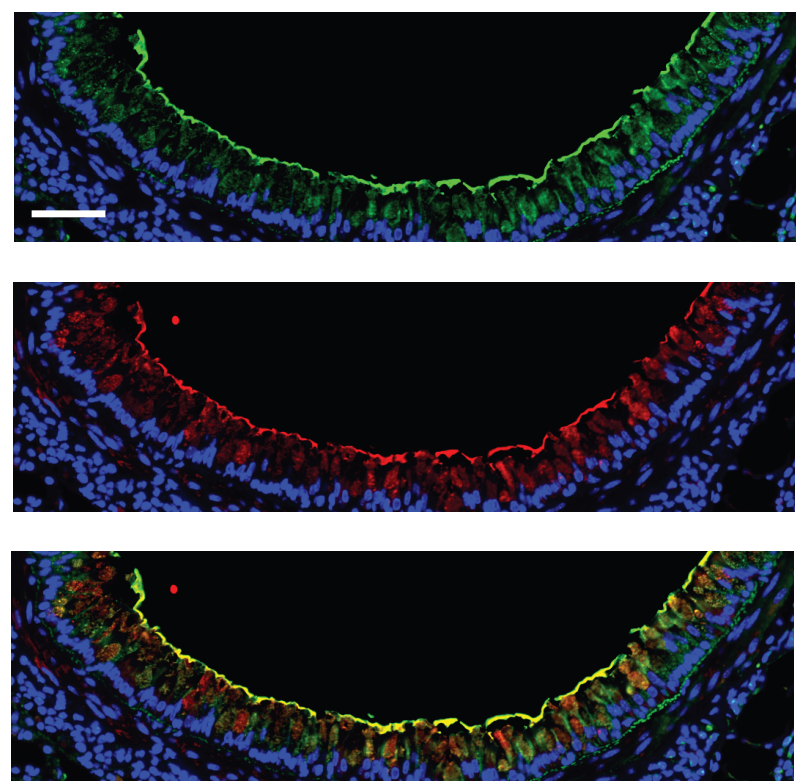

Figure 3: BPIFB1 and MUC5B colocalize in murine airway epithelial cells.

(A) BPIFB1 (green) and MUC5B (red) immunostaining in airway epithelial cells from allergen challegned mice. Scale bar $=10 \mu \mathrm{m}$. (B) Percentage of cells positively stained for BPIFB1 only, MUC5B only, or both in allergen challenged mice. $n=7$ mice. (C) BPIFB1 (green) and MUC5B (red) immunostaining in large airways of allergen challegned mice demonstrating BPIFB1 staining in luminal mucus. Scale bar $=50 \mu \mathrm{m}$. 
A

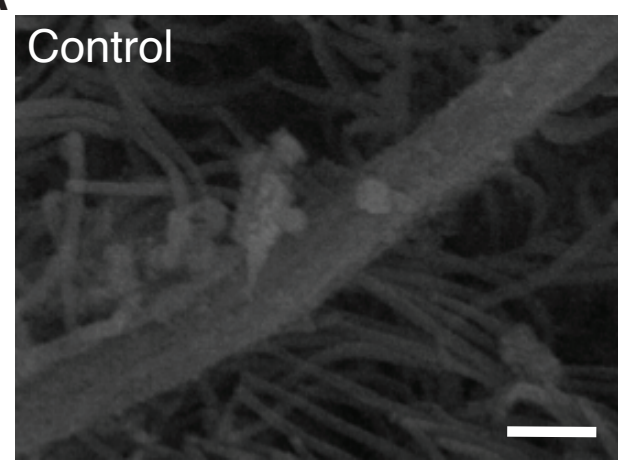

B

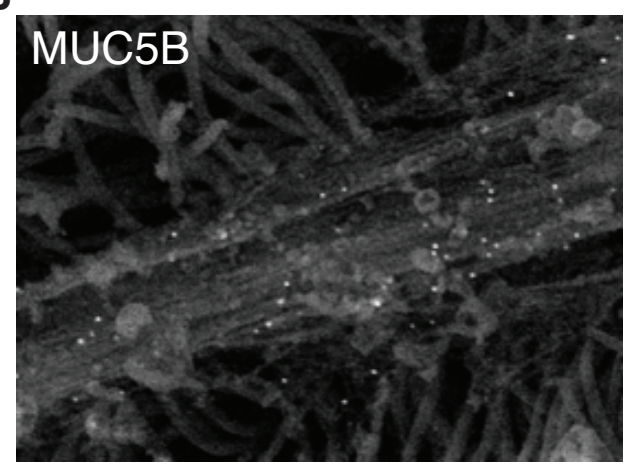

C

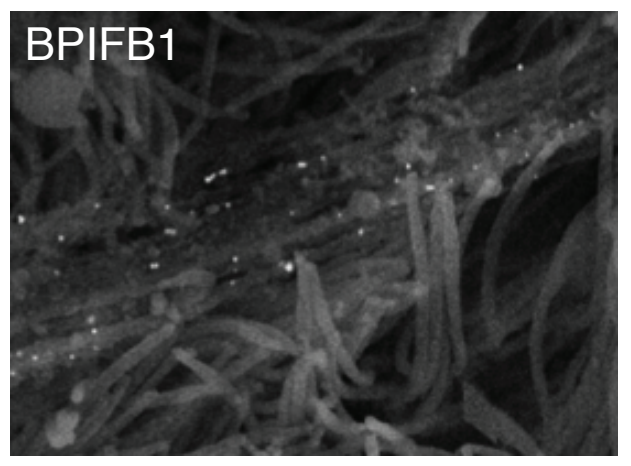

Figure 4: BPIFB1 is part of mucus netwrok in human bronchial epithelial cell culutures. Immunogold-labeled scanned electron microscopy for (A) negative control, (B) MUC5B, and (C) BPIFB1. Scale bar $=1 \mu \mathrm{m}$. 